\title{
High Precision Synchronization of a Large-scale Microwave Network over Stabilized Fiber Links
}

\author{
Wenting Wang ${ }^{1}$, Aram Kalaydzhyan ${ }^{1,2}$, Kemal Şafak ${ }^{1,2}$, Ming Xin ${ }^{1,3}$, Michael Y. Peng ${ }^{3}$, Kwangyun Jung ${ }^{4}$, \\ Jungwon Kim ${ }^{4}$ and Franz X. Kärtner ${ }^{1,2,3}$

\begin{abstract}
${ }^{1}$ Center for Free-Electron Laser Science, Deutsches Elektronen-Synchrotron, Notkestrasse 85, Hamburg 22607, Germany
${ }^{2}$ Physics Department, University of Hamburg and the Hamburg Center for Ultrafast Imaging, Luruper Chaussee 149, 22761 Hamburg, Germany

${ }^{3}$ Research Laboratory of Electronics, Massachusetts Institute of Technology, Cambridge, Massachusetts 02139, USA

${ }^{4}$ School of Mechanical and Aerospace Engineering, Korea Advanced Institute of Science and Technology (KAIST), Daejeon 305-701, Korea *email: wenting.wang@desy.de
\end{abstract}

\begin{abstract}
We demonstrate a high precision microwave network over stabilized multi-kilometer fiber links. Relative phase jitter $(>1 \mathrm{~Hz})$ and drift $(<1 \mathrm{~Hz})$ between two remotely synchronized 10.83-GHz microwave sources are 77.9 and $119.6 \mu$ rad, respectively, over 2.5-hour operation.

OCIS codes: (060.2360) Fiber optics links and subsystems; (060.5625) Radio frequency photonics; (120.5050) phase measurement
\end{abstract}

\section{Introduction}

High-precision long-distance time and frequency dissemination has attracted considerable attention in many applications including X-ray free-electron lasers [1] and ultra-stable clock transfer. In particular, a large-scale synchronized microwave network is required in radio telescope arrays [2], such as Atacama Large Millimeter Array and Square Kilometer Array, to achieve ultralow relative phase errors among many antennas over multi-km distance as well as in accelerators and light sources. Synchronization of these microwave sources using electrical phase detectors in RF domain has already reached a timing limit of 50-100 fs, which may become a potential obstacle for future ultra-high-resolution distant cosmic imaging. On the other hand, a mode locked laser (MLL) has an inherent advantage to simultaneously provide low jitter time markers and generate high performance microwave signals encoded in its repetition rate; it is therefore a promising candidate to serve as a timing reference for all antennas in this microwave network. In the past decade, we have developed a high precision timing distribution system using MLLs and balanced optical cross-correlators (BOC) [3] and demonstrated 1-fs precision local optical-to-microwave synchronization using balanced optical-microwave phase detectors (BOMPD) [4]. In this paper, we remotely synchronize two independent microwave sources over a 4.7-km fiber link network. The overall residual timing jitter is below $2 \mathrm{fs}$, which may significantly expand the exploration range of radio telescope arrays.

\section{Experimental Setup}

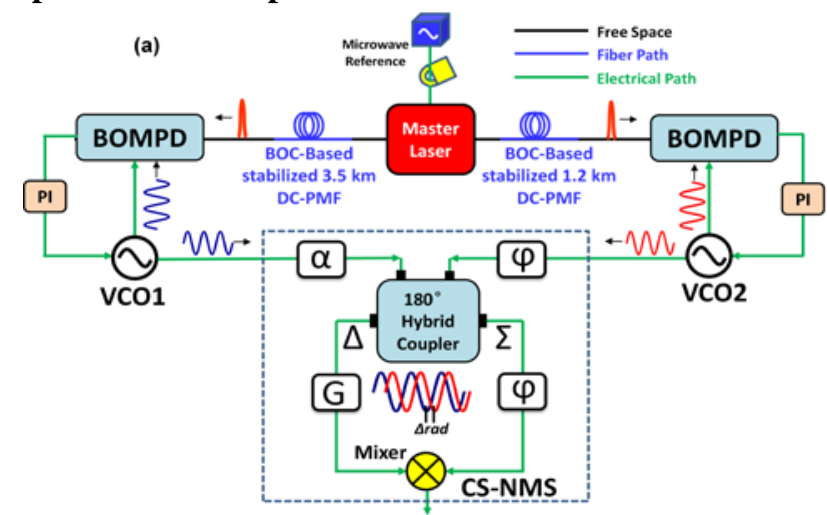

(b)
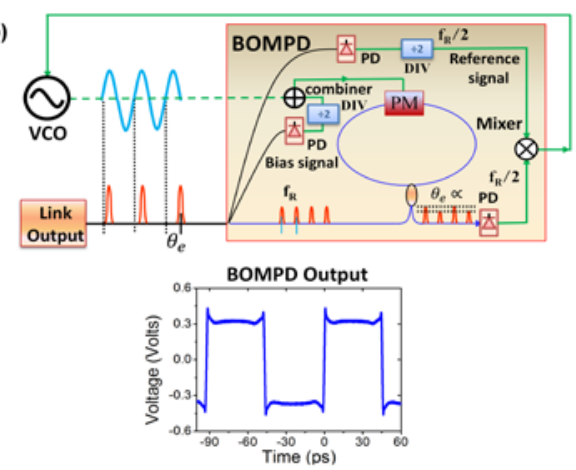

Fig. 1: (a) Experimental setup of the remote microwave synchronization network. (b) Schematic for an optoelectronic phase locking loop to synchronize a VCO to a remote timing signal. BOC: balanced optical cross correlator; BOMPD: balanced optical-microwave phase detector; VCO: voltage-controlled oscillator; DC-PMF: dispersion compensation polarization-maintaining fiber; $\varphi$ : phase shifter; $\alpha$ : attenuator; G: amplifier; PI: proportional-integral controller; PD: photodetector; PM: phase modulator; DIV: divider; inset is a transfer curve of BOMPD.

A diagram of the experimental setup is shown in Fig. 1 (a). The experimental setup contains four sections: master laser stabilization, dual timing link stabilization, remote microwave source stabilization, and carrier suppression phase noise measurement. The master laser is a MLL with a pulse duration of $160 \mathrm{fs}$ and pulse repetition rate of 216.667 MHz, which is phase locked to a microwave reference with a locking bandwidth of $10 \mathrm{~Hz}$ to enhance its 
long-term stability. The optical pulse train emitted from the MLL is split into two parts and then delivered as the timing signal into two independent dispersion compensated polarization maintaining fiber (DC-PMF) links with a length of $1.2 \mathrm{~km}$ and $3.5 \mathrm{~km}$, respectively. A fiber-coupled partial reflector at the end of each link provides optical signals for the BOCs in the link inputs upon back-reflection and for the BOMPDs in the link outputs upon transmission. The reflected pulses are combined with new laser pulses in the BOCs to generate error signals for active stabilization of the links to obtain a high precision timing reference at the end of each link. Each transmitted pulse is sent into a BOMPD to phase lock a remote voltage-controlled oscillator (VCO) operating at $10.833 \mathrm{GHz}$. The microwave signal at the output of each VCO is split into two parts: one part is injected into the BOMPD, and the other part is fed into the out-of-loop carrier suppression noise measurement setup (CS-NMS). Sensitivities of the two BOMPDs at the output of the $1.2 \mathrm{~km}$ link and the $3.5 \mathrm{~km}$ link are $0.6 \mathrm{mV} / \mathrm{fs}$ and $0.2 \mathrm{mV} / \mathrm{fs}$, respectively. We employ free space components to replace fiber components as well as shorten the fiber length of Sagnac interferometers (SGI) in the BOMPDs to optimize the long-term stability. As shown in Fig. 2 (b), optical pulses from the link output are detected by two free-space photodetectors (PD) to generate bias and reference signals divided by the frequency dividers (DIV) for BOMPDs. The generated error signal is sent into a proportional-integral (PI) controller, whose output is feedback to the VCOs to lock them remotely to the master laser. Then, the out-ofloop CS-NMS with noise floor of $69.7 \mu \mathrm{rad}$ and sensitivity of $23.2 \mathrm{~V} / \mathrm{rad}$ is used to measure the relative phase error between the two VCOs. In this CS-NMS scheme, attenuator $(\alpha)$ and phase shifter $(\varphi)$ are tuned to minimize amplitude and phase difference between the two microwave signals. A $180^{\circ}$ hybrid coupler acts as a microwave interferometer achieving constructive $(\Sigma)$ and destructive interference $(\Delta)$. The carrier-suppressed signal from the $\Delta$ output is amplified by a low noise amplifier (G) and converted to a baseband error signal upon mixing with the $\Sigma$ output. Phase shifter $(\varphi)$ in one of the arms of the mixer is used to set the two signals in quadrature ensuring pure phase detection.

\section{Results and Discussion}

Relative phase noise and drift between the two synchronized VCOs is measured by the out-of-loop CS-NMS. Fig 2 (a) shows the phase noise spectral density over the frequency range of [1 Hz, $100 \mathrm{MHz}]$. The accumulated phase error of the system above $1 \mathrm{~Hz}$ is only $77.9 \mu \mathrm{rad}$ for the $10.833 \mathrm{GHz}$ carrier frequency. The pink axis shows the equivalent SSB phase and it exhibits a phase noise of only $-108 \mathrm{dBc} / \mathrm{Hz}$ at $10-\mathrm{Hz}$ offset from the carrier. Fig. 2 (b) shows phase drift between two remotely synchronized VCOs below $1 \mathrm{~Hz}$. The timing deviation is only $8.85 \mathrm{fs}$ peak-to-peak and 1.76 fs RMS over 2.5 hours of continuous operation. The corresponding maximum phase deviation is about $602.7 \mu \mathrm{rad}$ and RMS phase drift is $119.6 \mu \mathrm{rad}$. These results indicate that the microwave network would enable few-fs level synchronization for radio telescope arrays, next generation accelerator facilities and light sources.

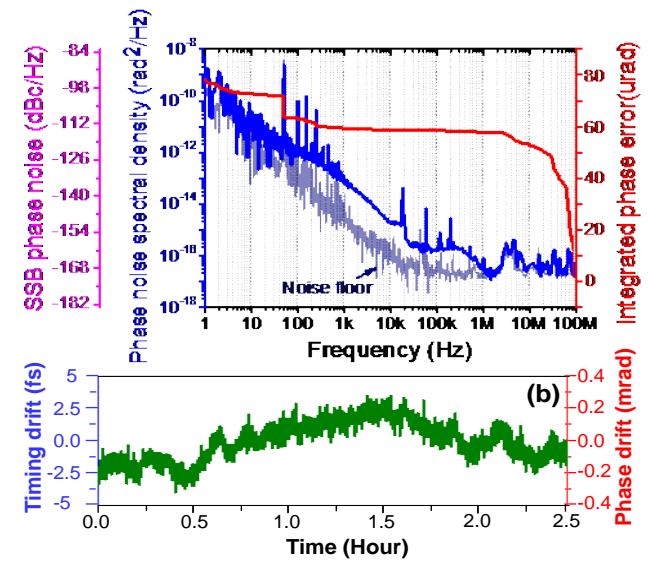

Fig. 2: Out-of-loop measurement results of the microwave network as detected by CS-NMS. (a) Measured phase noise spectral density, its corresponding integrated phase error at $10.833 \mathrm{GHz}$ carrier, and equivalent SSB phase noise; (b) out-of-loop timing and phase drift over 2.5 hours.

\section{References}

[1] J. Kim, et al., "Drift-free femtosecond timing synchronization of remote optical and microwave sources,” Nature Photonics 2, 733 (2008).

[2] J. Cliche, et al., "Precision timing control for radioastronomy," IEEE Control Systems Magazine, 2006.

[3] M. Xin, et al., "One-femtosecond, long-term stable remote laser synchronization over a 3.5-km fiber link,” Opt. Express 22, 14904 (2014).

[4] M. Peng, et al.,"Balanced optical-microwave phase detector for sub-femtosecond optical-RF synchronization,” Opt. Express 22, 27102 (2014).

\section{Acknowledgement}

W.T. Wang acknowledges the support by the China Scholarship Council to pursue graduate studies in Germany. 